\title{
ORIGINAL
}

\section{¿INFLUYEN LAS HUELGAS DE MÉDICOS EN EL PERFIL DE UTILIZACIÓN DE LOS SERVICIOS DE URGENCIAS HOSPITALARIOS?}

\author{
Francisco Buitrago, María José Gamero Samino, José María Vergeles Blanca y María Jesús \\ Cano Lozano. \\ Centro de Salud Universitario «La Paz» de Badajoz. Unidad docente de Medicina Familiar y Comunitaria.
}

\section{RESUMEN}

Fundamento: Valorar las diferencias existentes en el grado de adecuación de las consultas y en el perfil demográfico de la población atendida en un servicio de urgencias hospitalario durante el periodo de huelga de médicos de hospital de la primavera de 1995.

Métodos: Estudio observacional transversal en el Area sanitaria I de la provincia de Badajoz. Se incluyeron los 8.964 pacientes atendidos durante la huelga y los 8.024 atendidos en el mismo periodo del año 1994.

Resultados: La media de pacientes/día atendidos durante los períodos de huelga y control fue de $169,13 \pm 27,35$ y de $151,39 \pm 19,78(p<0.001)$, respectivamente.

Las características demográficas de los pacientes atendidos eran similares en ambos grupos estudiados, con iguales edades medias y distribución de sexos en los diferentes tramos etarios y también en los grupos obtenidos al hacer la desagregación de los pacientes por su lugar de residencia.

La mayoría de los pacientes acudieron por iniciativa propia $(70,1 \%$ y $65,8 \%)$ y $\sin$ ambulancia $(92 \%$ y $90,8 \%)$ en el período de huelga y control, respectivamente.

El alta domiciliaria fue el destino final de la actuación médica en el $85,35 \%$ de los pacientes durante la huelga y en el $83,81 \%$ en el Control, con tasas de hospitalización del $13,1 \%$ y $14,15 \%(\mathrm{p}<0.01)$, respectivamente.

Conclusiones: No se han encontrado diferencias importantes en el perfil de utilización del servicio de urgencias del hospital que puedan ser exclusivamente atribuibles a la huelga sanitaria.

Palabras clave: Urgencias hospitalarias. Huelgas médicas. Servicios de urgencia.
ABSTRACT

\section{Do Physicians Strikes Modify the Utilizacion profile of Hospital Emergency Facilities?}

Background:To assess the differences in appropriateness of consultations and demographic outline of people attended in a hospital emergency facility (HEP) along the hospital physicians strike period in spring 1995.

Methods: Observational cross-sectional study in Health Area $I$ in the province of Badajoz. 8964 patients assisted along the strike period were compared with 8024 attended in the same period of 1994 (no strike).

Results: The patients average was 169.13 (SD 27.35) a day in the strike period, during the control period this mean was 151.39 (SD 19.78) patients a day $(\mathrm{p}<0.001)$.

Demographic variables of patients were similar in both groups, with similar mean ages and gender proportion in all age and residence site groups.

Most of patients went to the HEF self-promoted $(70.1 \%$ and $65.8 \%$ ) and without ambulance $(92 \%$ and $90.8 \%$ ) in both periods (strike and control).

The outcome of medical care was home discharge in $85.35 \%$ during the strike period and $83.81 \%$ in the control period, with admission rates of $13.1 \%$ and $14.15 \%(\mathrm{p}<0.01)$.

Conclusions: There are no significant differences in the HEF use features completely explained by the phisicians strike.

Key words: Hospital Fmergencies. Physician Strikes. Emergency Facilities.
Correspondencia:

Francisco Buitrago Ramírez.

Sinforiano Madroñero, $\mathrm{n}^{\circ} 19-5^{\circ} \mathrm{A}$.

06011 BADAJOZ.

\section{INTRODUCCIÓN}

Las huelgas sanitarias de médicos de hospitales públicos suponen, de mantenerse un tiempo prolongado y con un alto grado de participación y seguimiento, conflictos sociales tan excepcionales que son situaciones interesantes para el es- 
tudio de la variabilidad de indicadores de proceso y resultados.

La sobrecarga asistencial que soportan los servicios de urgencias hospitalarios (SUH) es un tema ampliamente debatido y conocido, con un indudable origen multicausal. Repetidamente se señala que es la carencia y la deficiente calidad de la Atención Primaria de Salud la responsable, en parte, tanto del aumento de las demandas hospitalarias urgentes como de lo inadecuado de muchas de ellas ${ }^{1-6}$. Sin embargo, hasta un $80 \%$ de los pacientes acuden por decisión propia a los $\mathrm{SUH}^{7.8}$.Las barreras en la accesibilidad de los servicios de atención primaria, las listas de espera, la idolatría y expectativas hacia las nuevas tecnologías de uso hospitalario, el bajo umbral de tolerancia a la espera, la escasa educación sanitaria y la cercanía hospitalaria de la residencia habitual, son otros de los factores señalados como inductores de ese patrón de uso ${ }^{9-11}$.

Podría esperarse que situaciones como huelgas médicas prolongadas, con un seguimiento importante de los trabajadores, condicionasen una mayor y diferente utilización del SUH, pues la huelga conllevaría un aumento en la demora asistencial, con una mala aceptación social.

El presente trabajo se diseñó con el objetivo de conocer las diferencias habidas en el grado de adecuación de las consultas y en el perfil demográfico de la población atendida en el SUH durante la huelga médica de la primavera de 1995.

\section{PACIENTES Y MÉTODOS}

El SUH del Hospital Regional Universitario «Infanta Cristina» de Badajoz atiende las demandas de la población del área sanitaria I, con cabecera en la ciudad de Badajoz y un total de 216.635 habitantes. Además, como hospital regional, también recibe algunos pacientes trasladados desde los diferentes hospitales comarcales.

El estudio se realizó en el servicio de Urgencias-Admisión del mencionado hospital. Se analizaron la actuación médica y el destino final de todos los pacientes atendidos en el SUH durante la huelga sanitaria, que comenzó el 9 de mayo de 1995 y finalizó el 30 de junio del mismo año. Como grupo Control, para comparación de resultados, se eligió el conjunto de todos los pacientes atendidos durante el mismo período de ticmpo del año 1994 en el SUH.
Como fuente de datos se utilizó el registro de Admisión del SUH y se consideraron idóneas las consultas que finalizaron en ingresos hospitalarios, traslados a otro hospital, altas voluntarias o éxitus.

Los datos se procesaron en DBase-IV y los cálculos y pruebas de significación estadística (Chi cuadrado y «t» de Student) se realizaron con el programa Epi-Info (versión 6.02).

\section{RESULTADOS}

Un total de 8.964 pacientes acudieron al Servicio de Urgencias Hospitalario (SUH) durante el período de huelga sanitaria de 1995 , frente a los 8.024 que lo hicieron en el período similar del año precedente, lo que supuso un incremento del $11,7 \%$. Las características demográficas de las poblaciones atendidas se muestran en la tabla 1. La edad media fue de 41,96 y 41,46 años, con medianas de 39 y 38 y modas de 23 y 18 años, en los grupos de huelga y control, respectivamente.

La distribución de sexos es similar en los dos grupos, aunque con ligero predominio de varones en el número total de pacientes. La similitud en la distribución por sexos se mantiene en los diferentes grupos etarios, con predominio del sexo femenino en los mayores de 65 años.

Tabla 1

Caracteristicas demográficas generales

\begin{tabular}{|c|c|c|}
\hline & Período de huelga & Grupo control \\
\hline $\begin{array}{l}\text { Número total de } \\
\text { pacientes }\end{array}$ & 8.964 & 8.024 \\
\hline $\begin{array}{l}\text { Media diaria de } \\
\text { consultas }\end{array}$ & $169,1 \pm 27,4$ & $151,4 \pm 19,8^{* * *}$ \\
\hline Edad media (años) & $41,96 \pm 23,3$ & $41,46 \pm 23,2^{\mathrm{Ns}}$ \\
\hline $\begin{array}{l}\text { Porcentaje } \\
\text { varones/rnujeres }\end{array}$ & $53,8 \% / 46,2 \%$ & $53,6 \% / 46,4 \%{ }^{\mathrm{NS}}$ \\
\hline $\begin{array}{l}\text { Porcentaje de } \\
\geq 65 \text { años }\end{array}$ & $22,36 \%$ & $20,92 \% *$ \\
\hline $\begin{array}{l}\text { Porcentaje de } \\
\geq 30 \text { años }\end{array}$ & $38,40 \%$ & $39,13 \%$ Ns \\
\hline Porcentaje sin P-10 & $70,1 \%$ & $65,8 \% * * *$ \\
\hline $\begin{array}{l}\text { Porcentaje en } \\
\text { ambulancia }\end{array}$ & $8 \%$ & $9,2 \% * *$ \\
\hline
\end{tabular}

$\mathrm{NS}=$ No significativo.

$* \mathrm{p}<0.05$.

$* * \mathrm{p}<0.01$.

$* * * \mathrm{p}<0.001$. 
La mayoría de los pacientes acudieron por iniciativa propia al SUH, sin parte de interconsulta ni otro documento médico acreditativo de haber sido atendidos previamente en otro nivel asistencial. El porcentaje fue menor $(65,8 \%)$ en el período de control frente al de huelga sanitaria $(70,1 \%, \mathrm{p}<0.001)$.

El porcentaje de pacientes que llegaron en ambulancia fue $8 \%$ y $9,2 \%$ durante la huelga y en el grupo de control, respectivamente.

El destino final de los pacientes se muestra en la tabla 2, con un porcentaje de altas a domicilio del $85,35 \%$ durante la Huelga y $83,81 \%$ en el Grupo Control $(\mathrm{p}<0.01)$. Las tasas de hospitalizaciones fueron del 13,10\% y $14,15 \%(\mathrm{p}<0.05)$ en ambos periodos, respectivamente.

Tabla 2

Destino final de las consultas atendidas

\begin{tabular}{|lcc|}
\hline & Periodo de huelga & Grupo control \\
\hline Altas a domicilio & $85,35 \%$ & $83,81 \%^{* *}$ \\
Hospitalizaciones & $13,10 \%$ & $14,15 \%^{*}$ \\
Traslado a otro & & \\
hospital & $1,12 \%$ & $1,54 *$ \\
Altas voluntarias & $0,33 \%$ & $0,37 \%^{\mathrm{Ns}}$ \\
Exitus & $0,10 \%$ & $0,13 \%^{\mathrm{Ns}}$ \\
\hline
\end{tabular}

NS $=$ No significativo

$* \mathrm{p}<0.05$.

** $\mathrm{p}<0.01$

Tabla 3

Características demográficas de la población atendida con residencia en la ciudad de Badajox

\begin{tabular}{|c|c|c|}
\hline & Periodo de huelga & Grupo control \\
\hline $\begin{array}{l}\text { Número total de } \\
\text { pacientes }\end{array}$ & $4.733(52,8 \%)$ & $4.258(53,1 \%)^{\mathrm{NS}}$ \\
\hline Edad media (años) & $38,03 \pm 22,4$ & $37,42 \pm 22,4^{\mathrm{NS}}$ \\
\hline $\begin{array}{l}\text { Porcentaje } \\
\text { varones/mujeres }\end{array}$ & $52,8 \% / 47,2 \%$ & $51,9 \% / 48,1 \%$ Ns \\
\hline $\begin{array}{l}\text { Porcentaje de } \\
\geq 65 \text { años }\end{array}$ & $16,73 \%$ & $15,61 \%{ }^{\mathrm{NS}}$ \\
\hline $\begin{array}{l}\text { Porcentaje de } \\
\geq 30 \text { años }\end{array}$ & $44,53 \%$ & $46,24^{\mathrm{NS}}$ \\
\hline $\begin{array}{l}\text { Porcentaje sin } \\
\text { P-10 }\end{array}$ & $85,2 \%$ & $84,1 \%{ }^{\mathrm{NS}}$ \\
\hline $\begin{array}{l}\text { Porcentaje en } \\
\text { ambulancia }\end{array}$ & $2,4 \%$ & $2,4 \%$ NS \\
\hline
\end{tabular}

NS $=$ No significativo
Los resultados obtenidos con la separación de los pacientes por su lugar de residencia, según que ésta fuese o no en la ciudad de Badajoz, se muestran en las tablas 3, 4, 5 y 6, apreciándose que sólo existen pequeñas diferencias en los dos grupos de población entre los períodos de huelga y control.

\section{Tabla 4}

Destino final de las consultas atendidas de la población con residencia en la ciudad de Badajoz

\begin{tabular}{|lcc|}
\hline & Período de huelga & Grupo control \\
\hline Altas a domicilio & $90,58 \%$ & $89,73 \%^{\mathrm{NS}}$ \\
Hospitalizaciones & $8,40 \%$ & $8,69 \%^{\mathrm{Ns}}$ \\
Traslado a otro & & \\
hospital & $0,57 \%$ & $0,96 \%^{\mathrm{NS}}$ \\
Altas voluntarias & $0,38 \%$ & $0,42 \%^{\mathrm{NS}}$ \\
Exitus & $0,06 \%$ & $0,19 \%^{\mathrm{NS}}$ \\
\hline
\end{tabular}

NS $=$ No significativo. $* \mathrm{p}<0.05$.

\section{Tabla 5}

Características demográficas de la población atendida con residencia fuera de la ciudad de Badajoz

\begin{tabular}{|c|c|c|}
\hline & Periodo de huelga & Grupo control \\
\hline $\begin{array}{l}\text { Número total de } \\
\text { pacientes }\end{array}$ & $4.231(47,2 \%)$ & $3.766(46,9 \%)^{\mathrm{Ns}}$ \\
\hline Edad media (años) & $46,37 \pm 23,5$ & $46,02 \pm 23,3^{\mathrm{NS}}$ \\
\hline $\begin{array}{l}\text { Porcentaje } \\
\text { varones/mujeres }\end{array}$ & $55 \% / 45 \%$ & $55,5 \% / 44,5 \%{ }^{\mathrm{NS}}$ \\
\hline $\begin{array}{l}\text { Porcentaje de } \\
\geq 65 \text { años }\end{array}$ & $28,67 \%$ & $26,92 \%$ Ns \\
\hline $\begin{array}{l}\text { Porcentaje dc } \\
\geq 30 \text { años }\end{array}$ & $31,53 \%$ & $31,09 \%$ vs \\
\hline $\begin{array}{l}\text { Porcentaje sin } \\
\text { P.10 }\end{array}$ & $53,1 \%$ & $45,2 \% * * *$ \\
\hline $\begin{array}{l}\text { Porcentaje en } \\
\text { ambulancia }\end{array}$ & $14,2 \%$ & $17,1 \% * * *$ \\
\hline
\end{tabular}

NS $=$ No significativo. ${ }^{* * *} \mathrm{p}<0.001$

Tabla 6

Destino final de las consultas atendidas de la población con residencia fuera de la ciudad de Badajoz

\begin{tabular}{|lcc|}
\hline & Periodo de huelga & Grupo control \\
\hline Altas a domicilio & $79,50 \%$ & $77,11 \%^{* *}$ \\
Hospitalizaciones & $18,34 \%$ & $20,31 \% *$ \\
Traslado a otro & & \\
hospital & $1,75 \%$ & $2,18 \%^{\mathrm{NS}}$ \\
Altas voluntarias & $0,28 \%$ & $0,31 \%^{\mathrm{Ns}}$ \\
Exitus & $0,12 \%$ & $0,08 \%^{\mathrm{NS}}$ \\
\hline NS $=$ No significativo. & ${ }^{*} \mathrm{p}<0.05$. & $* * \mathrm{p}<0.01$.
\end{tabular}




\section{DISCUSIÓN}

Las huelgas de los médicos de hospitales públicos representan conflictos excepcionales que, si son secundadas masivamente, deterioran el funcionamiento del hospital y colapsan muchos de sus servicios. Los medios de comunicación y los propios médicos comparten la apreciación de que estas situaciones conllevan un uso aún más pronunciado del SUH. Sin embargo, nuestros resultados muestran que, si bien existen diferencias estadísticamente significativas en los parámetros analizados en ambas situaciones (de huelga y normalidad), estas diferencias probablemente no sean ni clínica ni sociológicamente relevantes. El perfil de uso del SUH de nuestro hospital regional es prácticamente similar en los dos períodos. Las diferencias más llamativas aparecen entre los habitantes de dentro y fucra de la ciudad donde se ubica el hospital y estriban en que un alto porcentaje de habitantes urbanos acuden al SUH espontáneamente, sin volante médico de derivación, circunstancia menos frecuente en el medio rural ${ }^{12}$. De hecho, estas diferencias pueden ser inherentes a las características sociológicas y demográficas de ambas poblaciones, con menores tasas de envejecimiento y edades medias también menores en la población urbana.
El dato más relevante durante el período de huelga, comparado con su homólogo del año 1994. ha sido el incremento del $11,7 \%$ en el número total de consultas atendidas, con una media diaria de $169 \pm 27,4$ frente a $151 \pm 19,8$ $(p<0.001)$. Sin embargo este incremento, en el número total de consultas y en la media diaria de consultas, es uniforme y mantenido en la tendencia natural del año 1995 y en la de los dos años precedentes (figura 1). Por tanto sería arriesgado atribuir tal incremento al efecto de la huelga sanitaria. Así, la media diaria de consultas durante 1993 fue de $153,39 \pm 6,6$ frente a las $161,83 \pm 5,5$ de 1994 y las $175,16 \pm 8,37$ de $1995(\mathrm{p}<0.01)$.

Nuestro trabajo también revela el alto porcentaje de altas domiciliarias entre quienes acuden al SUH, y por ende la baja proporción de ingresos hospitalarios en ambos períodos $(14,22 \%$ y $15,69 \%$, durante la Huelga y el Control, respectivamente). Este bajo índice de ingresos es similar al encontrado $(15,1 \%)$ entre los pacientes portadores de un volante o derivación médica en un trabajo previo y no difiere, tampoco, del que se origina entre todos los pacientes atendidos en un $\mathrm{SUH}^{8,13-18}$. Los datos son más impactantes si consideramos que un $10-35 \%$ de los ingresos realizados desde los SUH son inadecuados ${ }^{14,15,19,20}$.

\section{Figura 1}

Evolución mensual de la media diaria de consultas en el Servicio de Urgencia Hospitalario en los últimos tres años.

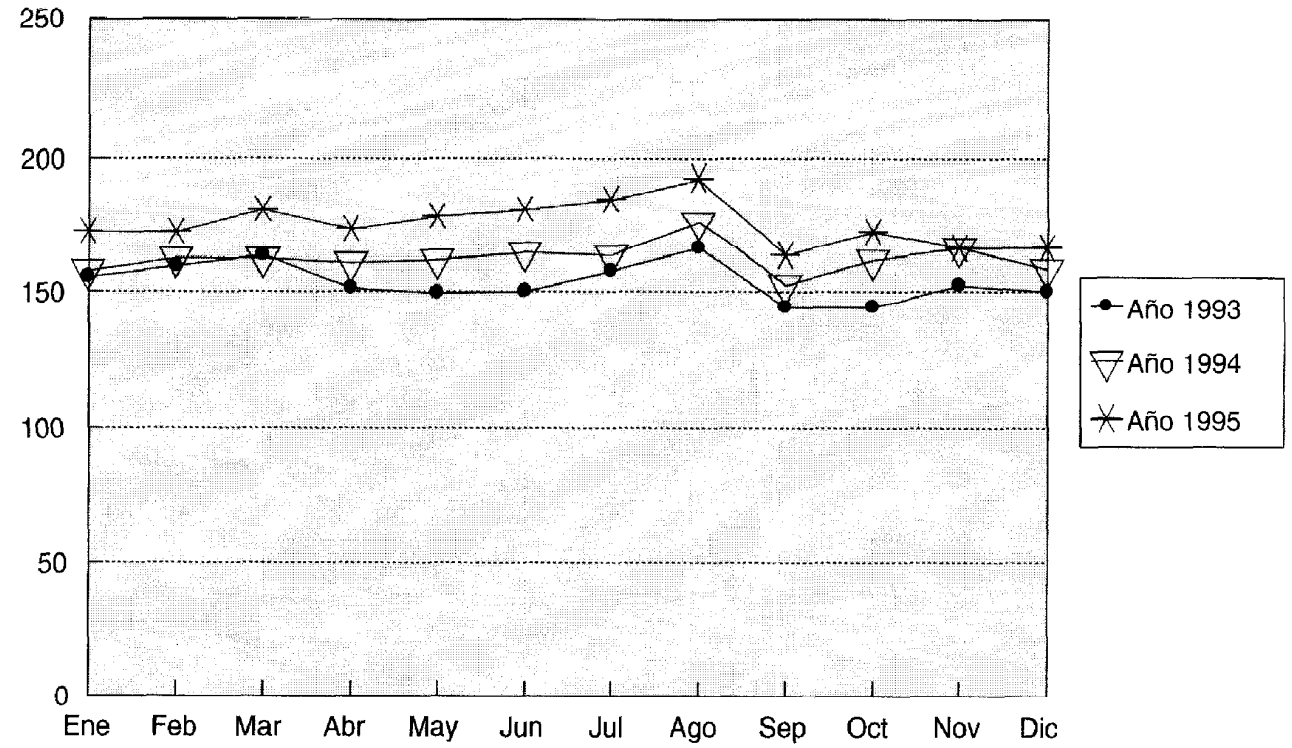


Evidentemente no todas las altas domiciliarias pueden considerarse consultas inadecuadas al SUH. En muchas de ellas se recurre a pruebas complementarias, a consultas con especialistas hospitalarios o a administración de terapéuticas no disponibles en Atención Primaria y que son necesarias en ese momento, sin que sea conveniente la demora en la asistencia o la asunción de su responsabilidad en otro nivel distinto del hospitalario.

Ampliando los criterios de idoneidad de las consultas al SUH, (incluyendo la permanencia en observación durante un tiempo $\geq 12$ horas y/o la petición de consulta con un especialista diferente al que recibe al paciente), no se encontraron tampoco diferencias entre ambos grupos de huelga y control ni entre éstos y los de un trabajo previo ${ }^{21}$, aunque el porcentaje de adecuación se incrementó obviamente al incluir estos criterios como definitorios de idoneidad de la derivación. Por eso, un protocolo que estandarizase y validase la adecuación de las derivaciones desde la atención primaria al SUH, de la misma manera que la Appropiateness Evaluation Protocol (AEP) ${ }^{22}$ hace con los ingresos hospitalarios ${ }^{16.20}$, podría ser de interés para homogeneizar y comparar los resultados de diferentes trabajos.

Sin duda la supravaloración social de la atención hospitalaria y el cambio de actitud frente a la enfermedad explican en parte la masiva y creciente utilización de los SUH, pero nuestro estudio revela que la huelga sanitaria, acaecida en la primavera de 1995 , no introdujo diferencias llamativas en el patrón de uso del SUH.

\section{AGRADECIMIENTOS}

Nuestro sincero agradecimiento al Sr. Manuel Murillo, del Servicio de Admisión del Hospital Regional Universitario «Infanta Cristina» de Badajoz, por su desinteresada e inestimable ayuda.

\section{BIBLIOGRAFÍA}

1. Lloret J, Puig X, Múñoz J. Urgencias médicas. Análisis de 18.240 pacientes atendidos en el servicio de urgencias de un hospital general en el período de un año. Med Clin (Barc) 1984; 83:135-141.

2. Román JL, Esnaola S. Estudio comparativo entre servicios de urgencia hospitalarios y atención primaria de salud. Med Integr 1982; 3:440-445.
3. Buitrago F, Peinado R, Merino J, Turégano JM. Análisis de las consultas urgentes en un centro de salud urbano. Aten Primaria 1991; 8:106-107.

4. Grupo de trabajo de la Sociedad Andaluza de Medicina Familiar y Comunitaria (SEMFYC). Ordenación de las urgencias en atención primaria. Aten Primaria 1992; 9:269-275.

5. Martínez Acevedo M, De Diego M.A., Del Río M, Ugartondo M.iLa saturación de las urgencias hospitalarias puede ser resuelta desde la atención primaria?. Aten Primaria 1993; 11:376.

6. Buitrago F.Atención continuada en los equipos de atención primaria: un tema pendiente. Aten Primaria 1991; 8:659-660.

7. Balanzó X, Pujol R y grupo Intercomarcal de Servicios de Medicina Interna. Estudio multicéntrico de las urgencias en hospitales generales básicos de Catalunya. Med Clin (Barc) 1989; 92:86-90.

8. Vilardell Ramoneda L.¿Por qué ingresan los paciente de forma urgente en un hospital?. Med Clin (Barc) 1994; 103:452-453.

9. Descarrega R, Gutiérrez C, Cruz L, López I.Análisis de la utilización inadecuada del servicio de urgencias de un hospital de tercer nivel. Aten Primaria 1994; 13:480-483.

10. Alberola V, Rivera F. La atención primaria como determinante de la utilización del servicio de urgencias hospitalario. Aten Primaria 1994; 14:825828.

11. Grumbach K, Keane D, Bindman A. Primary care and public emergency department overcrowding. Am J Public Health 1993; 83:372-378.

12. Rubio ML, Buñuel JC, Castillo JA, Palacín JC, Fernández A, Ruiz M.El P-10 como instrumento para la evaluación del uso de un servicio hospitalario de urgencias pediátricas por parte de la red de asistencia primaria. Aten Primaria 1992; 9:361-364.

13. González-Grajera B, Hormeño R, Buitrago F. Análisis de la cumplimentación de los nuevos modelos de derivación desde atención primaria a los servicios de urgencia hospitalarios. Aten Primaria 1995; 15:420-430.

14. Del Castillo M, Huguet J, Brabo J, Cortada L. Estudio del área de urgencias de un hospital general. Grado de adecuación de las visitas. Med Clin (Barc) 1986; 87:539-542.

15. Soler M, Verdaguer A, Mestre J, Vilatimó R. Dolor abdominal urgente. ¿Asistencia primaria o asistencia hospitalaria?. Med Clin (Barc) 1986; 87:175-178.

16. Muiño A, González VJ, Rodríguez de Castro E, Lázaro C, Fernández E.Asistencia en un servicio 
de urgencias: justificación de las visitas y adecuación de los ingresos. Rev Clin Esp 1988; 182:374378.

17. Martínez J, Portella E, De Pascual M.Razones del usuario en la utilización de un servicio de urgencias hospitalario. Rev Clin Esp 1992; 191:488493.

18. Rodríguez Artalejo F, González Montalvo JI, Sanz Segovia F, Jaramillo Gómez E, Banegas $\mathrm{JR}$, Rodríguez Mañas L et al. La urgencia geriátrica frente a la urgencia en la edad adulta: análisis retrospectivo de las urgencias médicas de un hospital general. Med Clin (Barc) 1989; 93:411-414.

19. Bañeres J, Alonso J, Antó JM. La adecuación de los ingresos hospitalarios. Med Clin (Barc) 1990; 95:357-358.

20. Matorras P, De Pablo M, Otero L, Alonso F, Daroca R, Díaz-Caneja N. Adecuación de los ingresos en un servicio de medicina interna de un hospital de tercer nivel. Med Clin (Barc) 1990; 94:290-293.

21. González-Grajera B, Mendoza R, Hinojosa J, Buitrago F.Adecuación de las derivaciones médicas a un servicio de urgencias hospitalario. Aten Primaria 1995; 16:433-436.

22. Gertmann PM, Restuccia JD. The appropriateness Evaluation Protocol.A technique for assessing unnecessary days for hospital care. Med Care $1981 ; 19: 855-870$. 